\title{
Propriedades psicométricas do Questionário de Avaliação de Desempenho no Trabalho em trabalhadores submetidos à radioterapia
}

\author{
Psychometric properties of the Brazilian version of the Work Role Functioning \\ Questionnaire evaluating workers in radiotherapy
Propiedades psicométricas del Cuestionario de Evaluación de Desempeño en el Trabajo junto a trabajadores sometidos a radioterapia

\author{
Cristiane Helena Gallasch'; Neusa Maria Costa Alexandre"I ; Sergio Carlos Barros Esteves ${ }^{\text {III }}$
}

\begin{abstract}
RESUMO: Afecções crônicas, incluindo neoplasias, representam sério problema de saúde pública por causarem incapacidade, absenteísmo e afastamento de trabalhadores. O estudo teve como objetivo avaliar as qualidades psicométricas do Questionário de Avaliação do Desempenho no Trabalho (WRFQ-Br) em trabalhadores com neoplasia submetidos à radioterapia no interior do Estado de São Paulo. Participaram do estudo 51 pacientes admitidos nos anos de 2011 e 2012, e 105 trabalhadores saudáveis. Na avaliação das propriedades psicométricas, observou-se alfa de Cronbach entre 0,77 e 0,93 na avaliação da consistência interna. Na avaliação da validade, houve diferença significativa nos itens que avaliam demandas de produção mental e social $(p<0,05)$ utilizando a técnica dos grupos conhecidos. Também houve correlação do escore total do WRFQ-Br com os índices da Escala de Karnofsky, na abordagem convergente. O WRFQ-Br demonstrou bons resultados na avaliação das suas propriedades psicométricas, sendo considerado confiável para utilização em trabalhadores com diagnóstico de câncer. Palavras-Chave: Neoplasias; reprodutibilidade dos testes; avaliação da capacidade de trabalho; enfermagem do trabalho.
\end{abstract}

\begin{abstract}
Chronic disorders, including cancer, are a public health problem, causing worker incapacity, absenteeism, resignation and dismissal. This study examined the psychometric properties of the Brazilian version of the Work Role Functioning Questionnaire (WRFQ-Br) in workers with neoplasms undergoing radiotherapy in up-state São Paulo. Psychometric properties were evaluated in 51 subjects with cancer diagnoses undergoing radiotherapy between 2011 and 2012, and 105 healthy workers. The results indicated good internal consistency (Cronbach alpha $=0.77-0.93$ ). Construct validity displayed significant differences $(p<0.05)$ between groups in output, and mental and social demands. Convergent validity analysis revealed a relation between the WRFQ-Br and the Karnofsky scale. Data showed that WRFQ-Br demonstrated reliable psychometric properties for evaluating workers with cancer diagnoses in Brazil.
\end{abstract}

Keywords: Cancer; reproducibility of results; work capacity evaluation; occupational health nursing.

RESUMEN: Enfermedades crónicas, incluyendo neoplasias, representan serios problemas de salud pública, puesto que causan discapacidad, absentismo y ausencias de los trabajadores. El objetivo de este estudio fue evaluar las propiedades psicométricas del Cuestionario de Evaluación de Desempeño en el Trabajo (WRFQ-BR) en trabajadores portadores de neoplasia, sometidos a radioterapia en el interior del Estado de São Paulo. Participaron en el estudio 51 pacientes admitidos en los años de 2011 y 2012 , y 105 trabajadores saludables En la evaluación de las propiedades psicométricas, se observó el alfa de Cronbach entre 0,77 y 0,93 en la evaluación de la consistencia interna. En la evaluación de validad, hubo una diferencia significativa entre los ítems que evalúan demandas de producción, mental y social $(p<0,05)$ utilizando la técnica de los grupos conocidos; y correlación del score total del WRFQ-Br con los índices de la Escala de Karnofsky, en el enfoque convergente. El WRFQ-Br ha mostrado buenos resultados en la evaluación de sus propiedades psicométricas y se considera fiable para utilización en trabajadores con diagnóstico de cáncer. Palabras Clave: Neoplasias; reproductibilidad de las pruebas; evaluación de capacidad de trabajo; enfermería del trabajo.

\section{INTRODUÇÃO}

Doenças crônicas como câncer, distúrbios osteomusculares, artrite, doenças cardíacas ou neurológicas representam um importante problema de saúde pública, ocasionando limitações funcionais e biopsicossociais, absenteísmo, afastamento das atividades de trabalho e reduzindo os índices de qualidade de vida ${ }^{1-5}$.
Atualmente, cerca de $12 \%$ das mortes registradas no mundo são relacionadas ao câncer, representando cerca de sete milhões de pessoas. São notificados onze milhões de casos novos de neoplasias malignas por ano, com previsão de elevação para 27 milhões até 2030, com 17 milhões de óbitos e 75 milhões de

${ }^{I}$ Enfermeira. Doutora em Enfermagem. Professora Adjunta do Departamento de Enfermagem Médico-Cirúrgica da Faculdade de Enfermagem da Universidade do Estado do Rio de Janeiro. Rio de Janeiro, RJ, Brasil. E-mail: cristiane.gallasch@gmail.com.

IIEnfermeira. Doutora em Enfermagem. Professora Associada da Faculdade de Enfermagem da Universidade Estadual de Campinas. Líder do Grupo de Estudos e Pesquisas em Saúde do Trabalhador e Ergonomia. Campinas, São Paulo, Brasil. E-mail: nmca@unicamp.br.

IIIRadio-oncologista. Doutor em Radiologia. Coordenador da Seção de Radioterapia do Hospital da Mulher Professor Doutor José Aristodemo Pinotti CAISM da Universidade Estadual de Campinas. São Paulo, Brasil. E-mail: estevesrt@uol.com.br.

${ }^{\text {IV } O s ~ a u t o r e s ~ a g r a d e c e m ~ o ~ a p o i o ~ d o ~ P r o g r a m a ~ d e ~ B o l s a s ~ d e ~ D o u t o r a d o ~ d a ~ C o o r d e n a c ̧ a ̃ o ~ d e ~ A p e r f e i c ̧ o a m e n t o ~ d e ~ P e s s o a l ~ d e ~ N i ́ v e l ~ S u p e r i o r . ~}$ 
pessoas vivas, segundos as estimativas da Organização Mundial da Saúde ${ }^{6}$. O aumento do número de casos novos relaciona-se diretamente ao aumento da expectativa de vida da população e à exposição a fatores de risco ${ }^{7}$. Aproximadamente, $50 \%$ dos casos ocorrem nos países em desenvolvimento, como o Brasil, onde houve uma estimativa de 576.000 novos casos em 2014 e $2015^{6,7}$.

O câncer, assim como outras doenças crônicas não transmissíveis, tornou-se frequente e cada vez mais comum, podendo levar instabilidade às famílias, quando o chefe da família adoece, sendo ele o provedor da única fonte de renda ${ }^{6}$. A sobrevivência ao câncer deve ser considerada de forma relevante no tratamento, uma vez que, devido ao aumento de sua incidência, é necessário prevenir os desafios que surgem frente a viver com diagnóstico de câncer. Esses sobreviventes completam um ciclo primário de tratamento e há a necessidade de seguir em frente com suas vidas ${ }^{8}$.

Limitações para o trabalho podem ser encontradas até 11 anos após o diagnóstico e início do tratamento9. A avaliação de pacientes com câncer encefálico, utilizando o Work Limitations Questionnaire (WLQ), encontrou restrições relacionadas à fadiga, depressão, ansiedade e perda cognitiva ${ }^{10}$. Sinais e sintomas semelhantes, exceto alterações cognitivas, foram encontrados em estudo envolvendo pacientes com diagnóstico de câncer torácico, mesmo quatro anos após diagnóstico e tratamento ${ }^{11}$. Sintomas residuais, após quimioterapia e radioterapia causam impacto direto no retorno ao trabalho ${ }^{12}$.

As pesquisas envolvendo as limitações no trabalho relacionadas ao tratamento e sobrevivência ao câncer são recentes, apresentadas na literatura internacional a partir da década de 2000. Os profissionais da área de saúde ocupacional e em oncologia precisam de informações e habilidades que permitam a realização de modificações no ambiente de trabalho e desenvolvimento de ações para melhoraria desses quadros e favorecimento do retorno ao trabalho ${ }^{10}$.

Considerando os resultados satisfatórios após a adaptação transcultural e avaliação da validade e confiabilidade do Questionário de Avaliação do Desempenho no Trabalho (Work Role Functioning Questionaire), adaptado para o Brasil (WRFQ-Br) para população com distúrbios osteomusculares, o objetivo deste estudo foi avaliar as propriedades psicométricas do questionário para trabalhadores com diagnóstico de câncer, submetidos à radioterapia, que mantiveram ou retornaram às atividades de trabalho durante ou após o tratamento.

\section{REVISÃo DE LITERATURA}

Pesquisadores e profissionais da área de saúde estão cada vez mais preocupados em utilizar escalas e questionários confiáveis e apropriados para determinada população ${ }^{13,14}$. A validade e a confiabilidade são particularmente importantes ao selecionar instrumentos que serão usados em pesquisas e na prática clínica.
No entanto, é importante ressaltar que estes dados não são qualidades estáticas de um instrumento. $\mathrm{O}$ ideal é reavaliar essas propriedades sempre que se mudar a população de estudo ${ }^{14,15}$.

A utilização de instrumentos validados tem sido considerada essencial para confiabilidade de dados obtidos junto aos indivíduos com dificuldades físicas ou alterações psicossociais. Dados confiáveis auxiliam no planejamento de ações de prevenção desses distúrbios e no planejamento de estratégias e programas de reabilitação na área da saúde do trabalhador.

O WRFQ-Br, com resultados satisfatórios de validade e confiabilidade, surge como potencial ferramenta para avaliação global do trabalhador com diagnóstico de câncer, considerando que um dos questionários que lhe deu origem já foi utilizado em estudo envolvendo câncer cerebral ${ }^{10,16}$.

Tendo em vista as recentes pesquisas que demonstram aspectos relacionados ao aumento da sobrevivência ao câncer e à necessidade de planejamento do retorno ao trabalho, durante e após o tratamento, essa população é apropriada para o desenvolvimento e aprimoramento de instrumentos de avaliação em saúde. Além disso, questionários relacionados à avaliação do desempenho no trabalho e sobrevivência ao câncer não estão disponíveis à população brasileira.

O referencial teórico utilizado neste estudo é a Psicometria, baseada nas normas de verificação e avaliação de propriedades psicométricas de instrumentos de medida estabelecidas pelo Consensus-based standards for the selection of health status measurement instruments (COSMIN) ${ }^{17}$.

\section{Metodologia}

Trata-se de um estudo metodológico, que segue as recomendações internacionais para avaliação das propriedades psicométricas do WRFQ-Br para indivíduos com diagnóstico de câncer, submetidos à radioterapia, de acordo com o referencial teórico citado.

Participaram do estudo dois grupos. $\bigcirc$ primeiro foi constituído de indivíduos com diagnóstico de câncer, submetidos à radioterapia em um centro privado especializado. E o segundo, composto por trabalhadores de um centro de desenvolvimento tecnológico sem queixas relacionadas à saúde, todos do interior do Estado de São Paulo.

Os critérios de inclusão para os sujeitos com câncer foram idade entre 18 e 75 anos, com diagnóstico confirmado em exame anatomopatológico, realização do protocolo de radioterapia, modalidade teleterapia, com exposição à dose mínima de 2000 cGy, término do protocolo de radioterapia há, no máximo, dois anos, manutenção das atividades de trabalho, formais ou informais, com carga horária mínima de 20 horas semanais ${ }^{16,18-21}$. Os mesmos critérios de idade, atividades de trabalho, 
carga horária semanal, além de ausência de queixas relacionadas à saúde foram considerados como critérios para inclusão dos trabalhadores saudáveis.

Foram considerados critérios de exclusão para os pacientes a realização de protocolo exclusivo de radioterapia na modalidade braquiterapia, e para ambos os grupos o analfabetismo.

Durante a coleta de dados, pacientes admitidos nos anos de 2011 e 2012 responderam ao WRFQ-Br de forma independente, no centro de atendimento em radioterapia, ou no domicílio, com formulários enviados via correio, de acordo com a opção do participante da pesquisa. Os trabalhadores sem queixas relacionadas à saúde preencheram os questionários no local de trabalho, de forma independente, sem influência dos profissionais do serviço de saúde ocupacional.

O protocolo de pesquisa foi submetido ao Comitê de Ética em Pesquisa da Faculdade de Ciências Médicas da Universidade Estadual de Campinas e obteve parecer favorável sob n¹04/2009, em 24 de novembro de 2009. Todos os participantes do estudo receberam e assinaram o termo de consentimento livre e esclarecido.

Os instrumentos utilizados e a análise de dados prevista são descritos a seguir e a discussão foi orientada pela literatura especializada ${ }^{1-17,22-33}$.

\section{Instrumentos de coleta de dados}

Foi utilizado um formulário, baseado em pesquisas anteriores ${ }^{16}$, adaptado especificamente para este estudo, para levantamento dos dados de identificação sociodemográfica e clínica. $\mathrm{O}$ tipo de trabalho foi descrito segundo a classificação de Hébert ${ }^{22}$.

O WRFQ originou-se dos instrumentos WLQ e Work Limitations - 26 Items (WL-26) ${ }^{21}$. O objetivo desses instrumentos consiste em avaliar se a capacidade para o trabalho está alterada devido a problemas de saúde, relacionados a afecções crônicas, causando impactos ou limitações nas atividades de trabalho diárias ${ }^{21,23}$. Em 2007, o WRFQ validado e os resultados confirmaram que os procedimentos para adaptação cultural deste instrumento para a versão brasileira na avaliação de indivíduos com distúrbios osteomusculares relacionados ao trabalho (DORT) foram realizados adequadamente ${ }^{16}$.

OWRFQ-Br inclui cinco subescalas, ou demandas de avaliação: plano de trabalho, avaliando dificuldades do trabalhador em planejar e manejar suas atividades ocupacionais diárias, do início ao término do dia de trabalho; demanda de produção, abrangendo produtividade, qualidade do serviço e satisfação no trabalho; demanda física, mensurando habilidade para executar atividades de trabalho, incluindo cargas físicas, dinâmicas e estáticas, como objetos pesados, movimentos, resistências, coordenação e flexibilidade; demanda mental, contemplando as exigências cognitivas relacionadas à atenção e à concentração; e demanda social, referindo-se às interações que o indivíduo deve realizar com seus colegas de trabalho e com os clientes de seu serviço ${ }^{16}$.
A KPS é um sistema de avaliação do estado clínico do paciente, que faz parte da consulta e registro sistemático, realizado pelos profissionais em saúde dos pacientes avaliados em oncologia. É utilizada para quantificar o bem-estar e as atividades da vida diária geral dos pacientes com câncer e permite que os pacientes sejam classificados quanto ao seu comprometimento funcional. Varia de 100 a 0, sendo 100-90 classificados como assintomáticos ou com sintomas mínimos, 89-70 sintomáticos, mas com capacidade de comparecimento ambulatorial, 69-50 permanência no leito pelo menos metade do dia, 49-30 permanecem no leito mais da metade do dia, 29. 10 acamados, com necessidade de cuidado constante e menor que 9 , paciente agônico ${ }^{24,25}$.

O WHOQOL-Bref, ou Whoqol-Abreviado, avalia aspectos gerais da qualidade de vida, incluindo demandas físicas, psicológicas, sociais e ambientais. Foi desenvolvido com base no WHOQOL-100, utilizado pela Organização Mundial de Saúde, e com resultados satisfatórios na avaliação de suas propriedades psicométricas para a população brasileira ${ }^{26}$.

O Índice de Capacidade para o Trabalho (ICT) é um questionário autoadministrado, utilizado na prática diária dos cuidados de saúde ocupacional, para avaliar a capacidade de trabalho de um indivíduo, por meio de sua percepção, com questões que englobam as exigências físicas e mentais de trabalho, o estado de saúde e os recursos do trabalhador ${ }^{27,28}$.

\section{Análise dos dados}

Os dados foram inseridos em banco de dados e analisados, descritivamente, por frequências absolutas (n) e relativas (\%) para as variáveis categóricas. Para as variáveis contínuas, foram utilizadas média, desvio-padrão, primeiro e terceiros quartis, valores máximos e mínimos. Para auxílio na organização, classificação e análise dos dados, foram utilizados os softwares computacionais Microsoft Office Excel 2007, SAS versão 9.2 - Cary, NC, USA: SAS Institute Inc., 2008 e R-project 2.15.0.

A confiabilidade foi avaliada por meio da consistência interna, que analisa se todas as subpartes de um instrumento ou de uma escala mensuram a mesma característica, e verificada por meio do coeficiente alfa de Cronbach 4,21,33,34. Foi estabelecido como evidência de consistência interna satisfatória valor de alfa de Cronbach $>0,70^{19,33-35}$. O procedimento foi realizado para cada questão em relação ao todo e para cada questão em relação ao domínio a que pertence.

A validade de constructo foi verificada por meio da abordagem dos grupos conhecidos, comparando os trabalhadores portadores de neoplasia com uma população de trabalhadores sem queixas relacionadas à saúde. Diferenças entre os grupos eram esperadas, podendo ser identificadas por meio dos escores ${ }^{18-20}$. Para as comparações entre os doentes e saudáveis, foi aplicado o teste não paramétrico de Mann-Whitney e para avaliação da validade convergente, o Coeficiente de Spermann, incluindo escores até 0,30 como satisfatórios, entre 
0,31 e 0,50 como moderados e, acima de 0,51, de forte magnitude ${ }^{28}$.

A validade convergente foi avaliada, correlacionando os escores obtidos na avaliação da população trabalhadora com diagnóstico de neoplasia utilizando os escores do WRFQ-Br, comparando-o com outros instrumentos que avaliassem os mesmos constructos, KPS, o ICT e o Whoqol-abreviado.

\section{Resultados}

\section{Caracterização dos sujeitos}

Participaram do primeiro grupo 51 sujeitos, com diagnóstico médico de neoplasia maligna, submetidos à radioterapia, usuários de um serviço ambulatorial privado de radioterapia do interior do Estado de São Paulo. O segundo grupo do estudo foi constituído por 105 sujeitos, sem queixas relacionadas à saúde, que foram selecionados entre trabalhadores de um centro de desenvolvimento de tecnologia. As características sociodemográficas desta população são descritas na Tabela 1 .

É observada diferença estatística significativa entre a média de idade dos grupos, com população mais velha entre os pacientes, o que era esperado quando se estuda câncer, encontrado mais comumente na população idosa. Ambos os grupos apresentam a maioria dos trabalhadores em atividades mistas de trabalho, característica já observada em estudos anteriores ${ }^{16}$. Não houve diferença estatística significativa entre os grupos na avaliação do número de horas trabalhadas por semana.
Os diagnósticos mais comuns de câncer entre o grupo de pacientes $(\mathrm{n}=51)$ foram de próstata $(23,53 \%)$ e câncer de mama $(45,10 \%)$. A dose médica de radioterapia, no momento da coleta de dados, foi de 5664cGy $(+117,1)$.

Com relação à Escala de Karnofsky, observaram-se escores de $80(19,61 \%), 90(17,65 \%)$ e $100(62,75 \%)$ entre os pacientes.

\section{Avaliação das propriedades psicométricas}

O instrumento apresentou adequados valores para consistência interna na avaliação da confiabilidade. $\bigcirc$ coeficiente alfa de Cronbach total obteve valor de 0,93, que indica alta precisão de medida. $\mathrm{Na}$ avaliação de cada subescala, observou-se maior precisão para plano de trabalho $(0,93)$ e menor para demanda física $(0,77)$.

A avaliação da validade de constructo foi realizada por meio da diferenciação entre os trabalhadores com diagnósticos de neoplasias submetidos à radioterapia e trabalhadores sem queixas relacionadas à saúde ou diagnóstico de câncer. Também pode ser denominada abordagem dos grupos contrastados, demonstrada na Tabela 2.

Foram observadas diferenças estatísticas significativas entre os grupos relacionadas às demandas de produção, mental e social. Não houve diferenças significativas relacionadas às demandas física e de plano de trabalho, assim como para o escore total. Os resultados dos instrumentos WRFQ-Br, KPS, Whoqol-Abreviado e ICT, utilizados para avaliação da validade convergente, são demonstrados na Figura 1.

TABELA 1: Características sociodemográficas de trabalhadores em radioterapia e indivíduos saudáveis. Campinas, SP, 2013. (N =162).

\begin{tabular}{lcc}
\hline \multicolumn{1}{c}{ Variáveis } & $\begin{array}{c}\text { Trabalhadores em } \\
\text { radioterapia } \\
(\mathbf{n}=51)\end{array}$ & $\begin{array}{c}\text { Trabalhadores } \\
\text { sem queixas } \\
(\mathbf{n}=105)\end{array}$ \\
\hline Gênero [f(\%)] & $23(45,1)$ & $46(43,8)$ \\
$\quad$ Masculino & $28(54,9)$ & $59(56,2)$ \\
Feminino & $53,4(10,1)$ & $34.8(11,8)$ \\
Idade média [anos(DP)] & $41.5(10.0)$ & $40.6(2.5)$ \\
Horas de trabalho semanais [média(DP)] & & $14(13.3)$ \\
Tipo de Trabalho [f(\%)] & $13(25.5)$ & $65(61.9)$ \\
Manual & $21(41.2)$ & $26(24.8)$ \\
Misto & $17(33.3)$ & 0.0663 \\
Não manual & & 20.001 \\
\hline
\end{tabular}

(*)Teste de Mann-Whitney

TABELA 2: Escores médios do WRFQ-Br em trabalhadores com câncer submetidos à radioterapia ( $\mathrm{n}=51)$ e em trabalhadores sem queixas relacionadas à saúde $(n=105)$. Campinas, SP, 2013.

\begin{tabular}{|c|c|c|c|}
\hline $\begin{array}{l}\text { Demandas } \\
\text { WRFQ-Br }\end{array}$ & $\begin{array}{l}\text { Trabalhadores submetidos à } \\
\text { radioterapia }\end{array}$ & $\begin{array}{c}\text { Trabalhadores sem } \\
\text { queixas }\end{array}$ & $p^{(*)}$ \\
\hline Demanda de plano de trabalho & $91,5( \pm 15,5)$ & $89,2( \pm 12,5)$ & 0.0183 \\
\hline Demanda de produção & $90,3( \pm 14,8)$ & $83,8( \pm 14,9)$ & 0.0003 \\
\hline Demanda física & $86,4( \pm 15,8)$ & $87,7( \pm 11,3)$ & 0.1551 \\
\hline Demanda mental & $89,0( \pm 16,6)$ & $86,1( \pm 13,7)$ & 0.0189 \\
\hline Demanda social & $94,3( \pm 11,5)$ & $90,9 \underline{( \pm 9,8)}$ & 0.0021 \\
\hline Escore total & $87,9( \pm 16,7)$ & $88,5( \pm 9,5)$ & 0.1599 \\
\hline
\end{tabular}

(*)Teste de Mann-Whitney 


\begin{tabular}{|lcc|}
\hline Correlação & Coeficiente de Spearman & p \\
\hline WRFQ-Br demanda plano de trabalho x KPS & 0,1901 & 0,01816 \\
WRFQ-Br demanda de produção x KPS & 0,2117 & 0,1359 \\
WRFQ-Br demanda física x KPS & 0,2877 & 0,0406 \\
WRFQ-Br demanda mental x KPS & 0,1175 & 0,4117 \\
WRFQ-Br demanda social x KPS & 0,1747 & 0,2250 \\
WRFQ-Br escore total x KPS & 0,3656 & 0,0083 \\
& & 0,0002 \\
WRFQ-Br demanda plano de trabalho x ICT & 0,4925 & 0,0001 \\
WRFQ-Br demanda de produção x ICT & 0,5078 & 0,0033 \\
WRFQ-Br demanda física x ICT & 0,4032 & 0,0053 \\
WRFQ-Br demanda mental x ICT & 0,3848 & 0,0048 \\
WRFQ-Br demanda social x ICT & 0,3923 & 0,0001 \\
WRFQ-Br Total escore x ICT & 0,5141 & $<0,0001$ \\
WRFQ-Br demanda física x Whoqol Físico & & 0,0014 \\
WRFQ-Br demanda mental x Whoqol psicológico & 0,6079 & 0,0388 \\
WRFQ-Br demanda social x Whoqol social & 0,4368 & 0,2932 \\
\hline
\end{tabular}

FIGURA 1: Correlações entre o WRFQ-Br com KPS, ICT e Whoqol-Abreviado aplicado nos sujeitos do estudo. Campinas, SP, 2013.

Na avaliação entre o WRFQ-Br e KPS, foram encontradas correlações com a demanda WRFQ- Br física e o escore total $(\mathrm{p}<0,05)$. Comparando o WRFQ-Br e o ICT, foram encontradas correlações entre todas as demandas e o escore total de ambos os instrumentos $(\mathrm{p}<0,05)$. O mesmo foi observado quando comparadas as demandas WRFQ-Br física $\mathrm{X}$ Whoqol físico, WRFQ-Br mental X Whoqol psicológico e WRFQ-Br Social X Whoqol social $(\mathrm{p}<0,05)$.

\section{Discussão}

O presente estudo teve início a partir da observação de queixas de pacientes submetidos à radioterapia afastados de suas atividades habituais de trabalho, que expressavam desejo de mantê-las, para vivenciarem a satisfação da produtividade, o convívio em seus círculos de amizade, e para poderem manter o pensamento em temas diferentes daqueles relativos ao tratamento e ao prognóstico da doença.

O retorno ao trabalho de indivíduos sobreviventes do câncer tem sido relatado como problemático, devido às alterações da capacidade física, fadiga, falta de apoio e adaptações no ambiente ocupacional, além da sobrecarga de trabalho ${ }^{36}$.

Desde o desenvolvimento industrial, com as mudanças na concepção sobre o trabalho e sua execução, os trabalhadores passaram a vivenciar longas jornadas, ritmos acelerados, fadiga física, divisões de tarefas e redução na participação do processo produtivo, o que resultou em alterações na saúde física e menta ${ }^{28}$.

Devido à alta exigência física e psíquica no trabalho, além das longas jornadas, tem sido observado grande número de afastamentos do trabalho na realidade do atendimento oncológico. Não há estatísticas da taxa de pacientes afastados entre todos os que apresentam a doença, porém, neste estudo, observou- -se 13,45\% dos casos em afastamento, mesmo após o término do protocolo proposto. Não são encontrados dados sobre a forma de avaliação da capacidade desses indivíduos ou de tentativas de adequação do trabalho, com redução de sobrecarga ou jornada, como formas de evitar o afastamento total das atividades laborais.

No Brasil, assim como observado em outras regiões do mundo, a elevação das taxas de sobrevida e também de cura após o tratamento em indivíduos em idade economicamente ativa aumentam as chances de permanência ou retorno às atividades de trabalho. Essa taxa de retorno varia de acordo com as características dos sistemas de suporte de cada país, incluindo os sistemas de saúde, reabilitação e de assistência sociail ${ }^{29}$.

Neste estudo, observou-se uma característica diagnóstica que segue a mesma tendência dos dados estatísticos brasileiros, com maior prevalência de câncer de próstata para homens e de mama para mulheres ${ }^{6}$. Neste grupo, a observação do elevado índice obtido na Escala de Karnofsky, com média de 94,31 (8,06), e a maioria dos pacientes apresentando escore $100(62,75 \%)$, é explicado pela inclusão de pacientes que mantinham atividades de trabalho neste estudo. Valores entre 100 e 90 indicam indivíduos que não apresentam sintomas ou estes são mínimos. Escores 89 e 70 demonstram que o paciente é sintomático, porém com capacidade de comparecimento ambulatorial para tratamento ${ }^{24,25}$. Isso indica que os indivíduos poderiam ter capacidade preservada para certas atividades, como trabalho. Ressalta-se que os valores obtidos indicam a média observada, porém a prática clínica utiliza apenas escores decimais na avaliação do estado de saúde (ex.: 70, 80, 90 ou 100).

\section{Avaliação das propriedades psicométricas}

$\mathrm{O}$ instrumento apresentou adequados valores para consistência interna. O Coeficiente alfa de Cronbach total obteve valor de 0,93 , que indica alta 
precisão de medida. Na avaliação de cada subescala, observou-se maior precisão para plano de trabalho $(0,93)$ e menor para demanda física $(0,77)$, porém ainda superior ao preconizado como satisfatório ${ }^{38,39}$.

O procedimento de adaptação cultural do mesmo instrumento para a população canadense com distúrbios osteomusculares apresentou menor valor de precisão para a demanda social $(0,66)$, com alfa de Cronbach maior de 0,80 para as demais subescalas ${ }^{21}$. Recentemente, o mesmo instrumento foi avaliado e validado para a população trabalhadora holandesa, com coeficiente alfa de Cronbach calculado entre 0,91 e 0,9636. O WL-26, questionário que deu origem ao WRFQ, obteve, durante seu processo de validação para portadores de doenças e lesões ocupacionais nos Estados Unidos, valores de alfa de Cronbach entre 0,88 e 0,924.

A correlação encontrada apenas entre a demanda física e a escala KPS é explicada pelo fato desta última referir-se a sintomas e sinais clínicos que remetem a desempenho físico. Não há menção a aspectos sociais, mentais ou atividades de trabalho ${ }^{25}$. A correlação encontrada entre o escore total do WRFQ-Br e a KPS sugere uma possibilidade de avaliação global dos pacientes com diagnóstico de neoplasia também por esse novo instrumento.

Na comparação entre o WRFQ-Br e o Whoqol-Abreviado, encontrou-se correlação com os domínios físico, psicológico e social do WRFQ-Br, com diferentes magnitudes entre os itens avaliados. Foi verificada correlação com a medida de qualidade de vida relacionada à saúde, o que fortalece o WRFQ-Br como fidedigno para avaliação desses aspectos.

O Whoqol-Abreviado já foi utilizado para avaliação de grupos com diferentes diagnósticos de câncer, com resultados confiáveis nestas populações. Mulheres submetidas à radioterapia no tratamento de câncer ginecológico, acompanhadas longitudinalmente, apresentaram a dor como aspecto que influenciou negativamente os domínios físico, psicológico e social ${ }^{37}$.

Mulheres submetidas a tratamento para câncer de mama, muitas vezes, experimentam mudanças nos comportamentos e modos de enfrentamento da doença e da vida, o que leva à busca por viver de forma produtiva e saudável, com iniciativas de retorno ao trabalho, atividades sociais, proximidade da família e envolvimento com crenças religiosas ${ }^{38}$. Alguns autores citam, ainda, que os trabalhadores com diagnóstico de câncer têm maior intervenção multidisciplinar para favorecer o retorno ao trabalho que a população em geral ${ }^{39}$.

Na comparação com o ICT, encontrou-se correlação com todas as demandas e com o escore total do WRFQ-Br, o que assegura fidedignidade na avaliação do desempenho do trabalhador. Quando agrupados segundo a categorização do ICT, os indivíduos apresentaram diferenças na demanda de produção e para o escore total do Questionário de Avaliação do Desem- penho no Trabalho. Isso é mais evidente entre aqueles categorizados em capacidade moderada e ótima.

A possibilidade de avaliação detalhada e com maior precisão da saúde do trabalhador, que recebeu o diagnóstico e foi submetido ao tratamento para neoplasias, pode favorecer o retorno ao trabalho, considerando aspectos econômicos, de produtividade e das relações interpessoais e sociais.

Com a realização de novas etapas de avaliação das propriedades psicométricas do WRFQ-Br para trabalhadores submetidos à radioterapia, sugere-se sua utilização para melhor avaliação das condições de saúde dos trabalhadores, das reais necessidades de afastamentos parciais ou totais, das adaptações necessárias para adequação do ambiente de trabalho e a implementação de programas efetivos de reabilitação e promoção da saúde do trabalhador.

Foi limitação do estudo a reduzida amostra $(\mathrm{n}=51)$, devido ao grande número de afastamentos das atividades de trabalho, de idosos atendidos (aposentadorias) e óbitos.

Há necessidade de estudos que acompanhem longitudinalmente esses pacientes, desde o diagnóstico inicial, havendo, assim, a possibilidade de avaliação do desempenho antes do afastamento, durante o tratamento e no planejamento das atividades e adaptações no retorno ao trabalho.

\section{Conclusões}

Os resultados do estudo confirmam que os procedimentos para avaliação das propriedades psicométricas do instrumento WRFQ-Br foram realizados com sucesso, de acordo com as recomendações da literatura internacional.

O instrumento apresentou alfa de Cronbach de 0,93 quando analisado integralmente, o que indica alta precisão de medida da consistência interna. Na avaliação da consistência interna de cada questão em relação à sua subescala, observaram-se valores entre 0,77 e 0,93.

Na avaliação da validade de constructo por meio da abordagem dos grupos conhecidos, houve diferença significativa nos itens que avaliam demandas de produção, mental e social $(\mathrm{p}<0,05)$. Não houve diferença significativa nas demandas física e social. A hipótese levantada e citada em algumas publicações é de que o trabalhador com diagnóstico de câncer recebe maior suporte multidisciplinar para o retorno ao trabalho. Questiona-se, ainda, se parte dessa população pode não diferir da população geral em alguns aspectos da avaliação em saúde.

Na avaliação da validade de constructo por meio de abordagem convergente, observou-se que o escore total do WRFQ-Br apresentou relação com os índices da Escala de Karnofsky, o que pode sugerir uma nova 
ferramenta para avaliação dos trabalhadores com câncer, mas, a partir de então, considerando aspectos globais dos indivíduos, e não apenas sintomas físicos.

Houve relação com todos os aspectos abordados pelo ICT e com os domínios físico, psicológico e social do Whoqol-Abrevidado, com diferentes magnitudes, reforçando a fidedignidade do Questionário de Avaliação do Desempenho no Trabalho para avaliação de aspectos funcionais do trabalho e da qualidade de vida dos indivíduos.

Assim, o WRFQ-Br demonstrou bons resultados na avaliação das suas propriedades psicométricas, sendo considerada confiável para utilização na população brasileira de trabalhadores com diagnóstico de câncer.

\section{REFERÊNCIAS}

1.Dall TM, Gallo P, Koenig L, Gu Q, Ruiz D. Modeling the indirect economic implications od musculoskeletal disorders and treatment. Cost Eff Resour Alloc. 2013; 11(5). Published online 2013 March 15. doi: 10.1186/1478-7547-11-5.

2.DiBonaventura MC, Gupta S, McDonald M, Sadosky A, Pettitt D, Silverman S. Impact of self-rated osteoarthritis severity in an employed population: Cross-sectional analysis of data from the national health and wellness survey. Health Qual Life Outcomes. 2012; 10(30). Published online 2012 March 15. doi: 10.1186/1477. 7525-10-30.

3.Steiner JF, Nowels CT, Main DS. Returning to work after cancer: quantitative studies and prototypical narratives. Psychooncology. 2010; 19(2):115-24.

4.Amick BC, Lerner D, Rogers WH, Rooney T, Katz J. A review of health-related work outcome measures and their uses, and recommended measures. Spine. 2000; 25:3152-60.

5.Feuerstein M. Introduction: The world challenge of work disability. J Occup Rehabil. 2005; 15:451-2.

6.Ministério da Saúde $(\mathrm{Br})$. Secretaria de Atenção à Saúde. Instituto Nacional de Câncer. Coordenação de Prevenção e Vigilância de Câncer. Estimativas 2008: Incidência de Câncer no Brasil. Rio de Janeiro: INCA: 2007.

7.Ministério da Saúde (Br). Secretaria de Atenção à Saúde. Instituto Nacional de Câncer. A situação do Câncer no Brasil. Rio de Janeiro: INCA: 2006.

8.Feuerstein M. Defining cancer survivorship. J Cancer Surviv. 2007; 1:5-7.

9.Yabroff R, Lawrence WF, Clauser S, Davis WW, Brwon ML. Burden of illness in cancer survivors: Findings from a population-based national sample. J Nat Cancer Inst. 2004; 96:1322-30.

10.Feuerstein M, Hansen JA, Calvio LC, Johnson L, Ronquillo JG. Work productivity in brain tumor survivors. J Occup Environ Med. 2007; 49:803-11.

11.Hansen JA, Feuerstein M, Calvio LC, Olsen CH. Breast cancer survivors at work. J Occup Environ Med. 2008; 50:777-84.
12. Tevaarwerk AJ, Lee JW, Sesto ME, Buhr KA, Cleeland CS, Manola J, et. Al. Employment outcomes among survivors of common cancers: the symptom outcomes and practice patterns (SOAPP) study. J Cancer Surviv. 2013; 7:191-202.

13.Marx RG, Bombardier C, Hogg-Johnson S, Wright JG. Clinimetric and psychometric strategies for development of a health measurement scale. J Clin Epidemiol. 1999; 52(2):105-11.

14.Selby-Harrington ML, Mehta SM, Jutsum V, Riportella-Muller R, Quade D. Reporting of instrument validity and reliability in selected clinical nursing journals. J Prof Nurs. 1994; 10(1):47-56.

15. Olivo AS, Macedo LG, Gadotti IC, Fuentes J, Stanton T, Magee DJ. Scales to assess quality of randomized controlled trials: a systematic review. Phys Ther. 2008; 88(2):156-75.

16.Gallasch CH, Alexandre NMC, Amick III BC. Cross-cultural adaptation, reliability and validity of the Work Role Functioning Questionnaire to Brazilian Portuguese. J Occup Rehabil. 2007; 17:701-11.

17. Mokkink LB, Terwee CB, Patrick DL, Alonso J, Stratford PW, Knol DL, et al. The COSMIN checklist for assessing the methodological quality of studies on measurement properties of health status measurement instruments: an international Delphi study. Qual Life Res. 2010; 19:539-49.

18.Mokkink LB, Terwee CB, Patrick DL, Alonso J, Stratford PW, Knol DL, et. al. COSMIN checklist manual. 2012. Amsterdan (BT): EMGO Institute for Health and Care Research: 2012.

19.Polit DF, Hungler BP. Fundamentos de pesquisa em enfermagem. $3^{\text {a }}$ ed. Porto Alegre (RS): Artes Médicas: 1995. 20.Dempsey PA, Dempsey AD. Using nursing research: process, critical evaluation and utilization. Philadelphia (PA): Lippincott Williams \& Wilkins: 2000.

21.Durand MJ, Vachon B, Hong QN, Imbeau D, Amick III BC, Loisel P. The cross-cultural adaptation of the Work Role Functioning Questionnaire in Canadian French. Int J Rehab. 2004; 27 (4):261-68.

22.Hébert F. Les indicateurs de lésions en santé et sécurité au travaiul: analyses par secteur d'activité économique em 1991. Montreaul (QC): Institut de recherche Robert-Sauvé en santé et sécurité au travail: 1996.

23. Amick III BC, Habeck RV, Ossmann J, Fossel AH, Keller R, Katz JN. Predictors of successful work role functioning alter carpal Tunnel release surgery. J Occup Environ Med. 2004; 46:490-500.

24.Simões JC, Gama RR, Winheski MR. Câncer estadiamento e tratamento. São Paulo: Lemar, 2008.

25.Karnofsky DA, Abelmann WH, Craver LF, Burchenal $\mathrm{JH}$. The use of nitrogen mustard in the palliative treatment of cancer with particular reference to bronchogenic carcinoma. Cancer. 1948;1:634-56.

26.Fleck MPA, Louzada S, Xavier M, Chachamovich E, Vieira G, Santos L, et al. Aplicação da versão em português do instrumento abreviado de avaliação da qualidade de vida 'WHOQOL-bref'. Rev Saude Publica. 2000; 34(2):178-83. 
27.Zwart BCH, Frings-Dresen MHW, Duivenbooden JC. Teste-retest reliability of the Work Ability Index. Occup Med. 2002; 52:177-81.

28. Mendes AMB. Aspectos psicodinâmicos da relação homem-trabalho: as contribuições de B. Dejours. Psicologia ciência e profissão. 1995; 15:34-38.

29.Rick O, Kalusche EM, Dauelsberg T, König V, Korsukéwitz C, Seifart U. Reintegrating cancer patients into the workplace. Dtsch Arztebl Int. 2012; 109(42):102-8. 30.Tamminga SJ, de Boer AGEM, Bos MMEM, Fons G, Kitzen JJEM, Plaisier PW, et. al. A Hospital-based work support intervention to enhance the return to work of cancer patients: a process evaluation. J Occup Rehabil. 2012; 22:565-78.

31.Roelen CAM, Koopmans PC, Schellart AJM, van der Beek AJ. Resuming work after cancer: a prospective study of occupational register data. J Occup Rehabil. 2011; 21:431-40.

32.Nunnaly JC. Psychometric theory. New York: McGraw-Hill: 1978.

33.Martins GA. Sobre confiabilidade e validade. Revista Brasileira de Gestão de Negócios. 2006; 8(20):1-12.
34.Pagano M, Gauvreau K. Princípios de Bioestatística. São Paulo: Editora Thomson; 2004.

35.Groeneveld I, de Boer AGEM, Frings-Dresen MHW. A multidisciplinary intervention to facilitate return to work in cancer patients: intervention protocol and design of a feasibility study. BMJ Open. 2012; 2:1-7.

36.Abma FI, van der Klink, Bültmann U. The Work Role Functioning Questionnaire 2.0 (Dutch version): examination of its reliability, validity and responsiveness in the general working population. J Occup Rehabil. 2013; 23 (1):135-47. 37.Vaz AF, Conde DM, Costa-Paiva L, Morais SS, Esteves SB, Pinto-Neto AM. Quality of life and adverse events after radiotherapy in gynecologic cancer survivors: a cohort study. Arch Gynecol Obstet. 2011; 284:1523-31. 38.Regis MF, Simões MFS. Diagnósticos de câncer de mama, sentimentos, comportamentos e expectativas de mulheres. Rev Eletron Enferm. 2005; 7(1):81-6.

39.Boer AGEM, Taskila TK, Tamminga SJ, Frings-Dresen MHW, Feuerstein M, Verbeek JH. Interventions to enhance return-to-work for cancer patients. Cochrane Database of Systematic Reviews 2011, Issue 2. Art. n: CD007569. DOI: $10.1002 / 14651858 . C D 007569 . p u b 2$. 